DOI: 10.2478/RAE-2019-0011 Review of Artistic Education no. 172019 106-112

\title{
11. EXTRAMUSICAL ASPECTS REGARDING THE ORGANISATION OF CHILDREN SYMPHONY ORCHESTRA
}

Ciprian Ion ${ }^{120}$

\begin{abstract}
This article introduces the problems that could emerge when undertaking to organize a children symphony orchestra, but also the means of surmounting them. The stress falls on the difficult mission of establishing the specific hierarchy (part master, placement on desks, choosing the concert-maestro), action that proves to be extremely delicate taking into consideration the age category we refer to. Moreover, we pursue the indication of the stages that should be taken into account in order to obtain an independent, functional and adaptable ensemble, given the limited time period until the public show. After choosing the members of the hard core of the ensemble, it is recommended that the master shall empower and present the tasks that one must meet. Thus, the qualities that the chosen children should take into consideration for the purpose of avoiding certain stressed moment or certain syncopes for the optimal operation of the orchestra are presented. Once the premises for a good operation have the created, the professor can transfer certain tasks to the students (tuning, settling the bows, part study, etc.) having as an effect the efficiency of going through the programme under the conditions of a deadline which cannot be exceeded. Furthermore, several recommendations regarding the psychological training of the children for the purpose of surmounting the stress factor specific to any public interpretation are presented.
\end{abstract}

Key words: orchestra, children, hierarchy, ensemble, psychology

\section{Introduction}

The famous novel "Lord of the Flies" by William Golding suscitates a long series of controversies regarding the existence of children innocence. The psychological issue, exposed in the form of an ample narrative, is extremely complex, however, an apparently minor aspect caught our interest, taking into account the fact that we have had the experience of leading a children orchestra for seven years. From the large group of children stranded on an island, only those who were part of a choir managed to impose themselves, to reorganize into a well-articulate, and unfortunately, authoritarian structure. This detail, insignificant to an outside reader, was the starting point for this research, endeavouring to explain the manner in which the hierarchical organization of any musical ensemble would be achieved in a correct manner.

The symphony orchestra represents one of the most complex forms of inter-human collaboration that has goal the acoustic materialization of compositions composed for this ensemble. The main problems emerging in the performance of this artistic undertaking are synchronization up to the stage of

\footnotetext{
${ }^{120}$ Associate Professor PhD., "George Enescu" National University of Arts from Iaşi, Romania, email: andicip@yahoo.com
} 
homogeneity at the technical and emotional level, obtaining a valuable artistic products under stress conditions (being in a flap due to interpretation in front of an audience) and the capacity to solve potential problems which could arise throughout interpretation. The goal must be cohesion, that "bi-dimensional construct made up of the cohesion which entails the task (the degree in which the members are engaged and support the performance of the task) and social and emotional cohesion (includes both interpersonal relations between individual and the pride to be a member of the same group)." (Onici, 2014, p. 161). Without pushing it, we can make comparisons between the manner of organization and operation of an orchestra with any other band which entail a collective effort, from completely different fields of activity: a football team, a military squadron, all needing organization for the purpose of synchronizing and obtaining the desired outcomes.

\section{Establishing hierarchy in the orchestra}

This article wishes to put in the limelight especially extra-musical problems regarding the manner of organizing symphony orchestra made up of children. If a conductor, who works especially with professional ensembles, has the role of dealing only with the artistic side, the teacher conductor must make an additional effort, sometimes much more arduous, regarding the structure of the orchestra in a universally accepted hierarchical form. We undoubtedly refer to establishing a maestro concert, of part leaders, but also of the place that each student must have at the desk depending on his/her musical experience, reliability, implication and the good cooperation with his/her colleagues.

The creation of this hierarchy must not give rise to any discontentment, as any wrong assessment of suspicion shall directly reflect on the acoustic quality of the ensemble. Most of the times, children know how to self-assess, know exactly the place they deserve or not to have, know how to accurately choose their leaders. It is self-explanatory that many of them shall want to have a more privileged place in the orchestra hierarchy, however, there is a sort of tacit collective reason that prevent them, in a subliminal manner, to accede to a position that they are aware do not deserve. This reference of each member of the orchestra should be well monitored, taking into account that some students may be motivated towards occupying a more important place, as others can be discourages, aspect that is not in the slightest desired. Any intervention in this self-organization should be characterised by diplomacy and be very wellreasoned.

If regarding the artistic side of the activity, the conductor must impose his/her view, the same thing is not recommended in the case of the manner of organizing the ensemble. One of the most important characteristics of an orchestra, regardless of the age of its members, is its capacity to exist and perform under the baton of a conductor. We do not consider the absolute dependency of the mass on only one person to be beneficial, these types of 
constructions being rigid, unadaptable to new and ephemeral. The main argument in this regarding would be that any authoritarian intervention creates emotional imbalance and inhibits creativity, lack of diplomacy or politeness being the reason for certain fissures with palpable repercussions at the level of sonority. The situation becomes even more serious taking into account the early age of the members of the orchestra. It is obvious that building an independent and flexible ensemble with a large group of children or youngsters represents an arduous and long-term work.

In the case of friction throughout the establishing of part leaders the intervention of the teacher conductor in a transparent manner that would involve in a discrete manner even the members of the orchestra is necessary. One must insist on the fact that it is not necessarily that soloistic virtuosity shall prevail for the purpose of occupying such a position, but a composite of qualities that have a secondary role within the classes of the main instrument. Thus, the so-called auditions for the purpose of establishing the part leaders can have wrong results, as these highlight only the interpretative virtuosity, certainly, extremely important, but insufficient in relation with the tasks that the student or youngster who shall occupy that position must have. For this purpose, it is necessary that we demarcate musical qualities of extra-musical ones.

The main musical quality (except for the solid mastery of instrumental technique) would be a good first glance reading. The easy performance of the moment of deciphering the musical text mainly means time gained for the purpose of channelling the interpretative effort towards solving problems. Moreover, the potential part leader should have a good harmonic hearing, even though the instrument that he masters is a monodic one. Singing in an orchestra entails not only knowing very well one's own esteem, but integrating, listening, following as much as possible from the text of one's colleagues from other parts.

Regarding extra-musical qualities, I would dare to start with the one of conductor. Basically, students have a tendency to follow a colleague's indications if they are pertinent, reasoned and presented in a delicate, not imperative manner. The proper part leader is the one who helps with the good carrying out of the rehearsal and not the one who could give rise to small timeconsuming conflicts. Taking into account that the teacher conductor and, implicitly, the entire ensemble, are always subjected to certain deadlines (the date of the concert) that are difficult to postpone, loss of time in rehearsals creates not only an emotional discomfort, but, in more serious cases, can affect the quality of the final product. Moreover, the part leader must have the capacity to create safety in the attack for the entire group, to be a bond between the wishes of the conductor and the technical possibilities of the colleagues.

Similarly delicate can become the placement at desks (here we refer, certainly, mainly to the string orchestra) as this must also be performed on a hierarchical basis. Starting from the first desk, the colleague of the part leader must be capable at any point to replace him/her in the unpleasant cases when 
he/she cannot be present. The good operation of the orchestra does not depend on the presence of a small group of performers, the safety measures for the purpose of avoiding unpredictable situations being imperative. We recommend the placement at desks having as a main criterion the experience in the orchestra, in our case the age criterion. Certainly, certain exceptions can be accepted, but in the case of student ensembles, where the time interval of experience is insignificant, any unjustified mess can have as an effect frustrations, which, by accumulation, generate smouldering conflict or the feeling of favouritism.

Another criterion that we must take into account is the reliability one, of attendance, of the degree of involvement. A large number of absences (justified or not) is impossible to recover in the case of ensembles, room or orchestral, as individual study can only remotely supply the gaps accumulated. Taking into account the degree of involvement in the placement at desks must not be perceived as a punishment, the conductor having the task of reasoning the decision of a "demotion", with the possibility of a rehabilitation in the case of a change in attitude. However, we recommend to not abuse such coercive measures, as they generate insecurity. Frequent changes of desk partners destroys the bond that is created in time between the children, thus, it would be ideal that the pair to not be modified for a larger period of time.

The most difficult and delicate task in organizing an ensemble is choosing the concerto maestro, who is the de facto leader of the orchestra. He/she must meet all the above-mentioned qualities, to enjoy the recognition of the colleagues and be a virtuoso, taking into account the countless works when he/she must perform difficult soloistic moments. Essentially, he/she must be a leader and an artist at the same time, the main stability factor, as the conductor can be changed, but the concerto maestro cannot. Such personalities usually come apart and the teacher must be careful not to intervene in a negative sense in the natural and unanimously accepted hierarchy.

After choosing the members of this hard core of the ensemble, it is recommended that the master shall empower and present the tasks that one must meet. Any leading position means very few privileges and a lot of work. The relationship between the conductor and the ones chosen shall be based on collaboration, trust, acceptance of solutions, awareness of the importance that it has. The complex organism that is represented by the orchestra cannot function in the absence of such a collaboration, any form of authoritarianism having as a result an artificial, uptight sonority, lacking sensitivity. "The main principles of teaching under conditions of cooperation establish a large variety of interaction forms, of episodic assistance of the deskmate (desk in our case) by the more advanced student, up to cooperative teaching groups organised by the teacher in terms of a certain educational technology." (Cosmovici, Iacob, 2008, p. 242)

We do not recommend the rotation of desks as a method of empowering all the members of the orchestra. Periodically bringing the last desks in front shall generate frustration and malicious hilarity, and, in the most serious cases, loss of 
trust and pleasure to attend the orchestra course. This shall anyway be done in time by the natural advance resulting from moving to a superior grade. Each year of experience shall offer assurance and assurance offers trust. The artificial intervention in the fair nature of this long journey could have as an effect losing the goal, and lack of trust in any field is a serious obstacle in professional accomplishment. Furthermore, any modification in the organic structure of the ensemble slows down the assimilation of information, which leads to meeting the performances desired in a longer period of time.

\section{Moments preceding rehearsal and the concert}

Once the problem of organizing the orchestra from a hierarchical point of view has been solved, time is necessary so that each department shall operate in optimal parameters, but also for the creation of connections between the parts. The first important moment, which precedes any rehearsal or concert, is carrying out the tuning. Except for the concentration of all the students' hearing, this undertaking actually represents the beginning of a complex mechanism in line with a well-established order. The teacher conductor must teach the orchestra to tune by itself, in a short amount of time. There is not didactic or musical argument for the tuning carried out by the conductor, with moment of insisting on a small group of instrumentalists or the entire ensemble. The effect become an undesired one, so that the desideratum of having a grip on the audience and the focus splash in a boring moment for the ones who are not involved in the process, and for the ones forced to seriously polish the instrument's sonority the effect shall be one of aggravation. Certainly, the prompt intervention of the conductor in cases of major slips is recommended. After a reasonable period of time, in which it is noticed that the tuning has become a skill for the students, the conductor can issue the natural demand to find the orchestra tuned and ready for starting the rehearsal future interpretative career, being the generally accepted tradition in all professional orchestras. It is possible that at the beginning the tuning without supervision to not be perfect due to various reasons, however, at the beginning of the rehearsal one can notice that even under these conditions, so that along the way it would adjust initial failures. Tuning again the entire orchestra, after it had tuned itself, represents a moment of didactic weakness, and not in the slightest, one of imposing oneself in front of the class, the gesture could be interpreted as a cancellation of the main requirement or a lack of trust regarding the children who endeavoured to carry it out. The ones who did not carry out the task very well shall understand the teacher's discontent, and the one of the colleagues, but, in time, shall improve themselves.

Highly important is the assimilation of the protocol during the concert: how to receive the conductor and, if applicable, the moment when the orchestra should stand up, who and when shakes the hand of the concerto maestro, etc. Complying with these traditions, century old, strike elegance, politeness and 
sobriety to the artistic action, but also constitutes a sort of psychological training for the collective artistic undertaking that shall be carried out under emotional stress conditions. Public interpretation, or individual one, either room or orchestral, is characterised by a specific feeling, sometimes with beneficial effects, other times with destructive ones. The tuning, except for its practical utility, can also be a moment when the children get used to the stage, the audience and the sonority of the hall, while the protocol moment, marked by the presence of the conductor, can give them trust, courage and the feeling that they have in front of them the person who trained and who shall lead them during the concert. The conductor's feelings should not be understated, aware of his/her responsibility, who through the standing up of the orchestra receives the role of a leader at the level of gestures (ideally would be for him/her to also receive fondness and empathy), and by shaking the hand of the concerto maestro, the transfer of tasks and feelings used in the artistic action shall be sanctioned. On the stage, we do not have to deal with relationships specific to the educational field (teacher-student), but with the ones specific between performers and conductor. Formal awkwardness, easily accepted for a children orchestra, creates however awkward scenes, thus a serious training of this moment is recommended.

The last rehearsals preceding the concert also have the role of a psychological training for the purpose of overcoming the thrills. Except for the last finishing actions regarding the musical aspect, the conductor can insist on the beautiful aspect of public interpretation, especially that the audience, in these cases, is empathic and committed to the ensemble. As a cheering factor one can understate the effect that potential awkwardness could cause, focusing on the general musical construction, on the pleasure of collective activity for the purpose of presenting the effort sustained throughout so many rehearsals.

\section{Conclusions}

Cultivating the sense of responsibility, respecting hierarchy (correctly carried out in advance) and the functionality of the parts, individually and together, give rise to an independent and flexible orchestra with which one could obtain musical performances in an optimal period of time. It is obvious that the teacher conductor also confronts with the drawback of generation changes, each year receiving novices while experimented instrumentalists move on to a superior level in their career. But there is always a core which can take over the teacher's didactic task, making his yearly repetitive work easier.

Returning to Golding's novel, we must take the warning sign of the entire narrative seriously, certainly, not that frightening level of seriousness. Basically, the small army that we build in the service of music must be permanently monitored, adjusted, motivated and encouraged, but, under no circumstance, must it be get out of hand. There are two safe ways of losing the authority in front of an ensemble: not getting involved and dictatorial behaviour. 


\section{References}

1. Boncu, Ș; Ceobanu, C (2013). Psihosociologie școlară, Ed. Polirom

2. Cosmovici, A.; Iacob, L (2008). Psihologie școlară, Ed. Polirom

3. Gâscă, N (1982). Arta dirijorală, Ed. Didactică și Pedagogică

4. Golding, W (2017). Impăratul muştelor, Ed. Humanitas

5. Onici, O (2014). Psihologia grupurilor școlare, Ed. Universității “Alexandru Ioan Cuza" 